\title{
Characteristics of technogenic soils developed from Neogene and Quaternary sediments substrate on reclaimed sulphur and sand extraction mine sites
}

\author{
Bartłomiej Woś, Marcin Pietrzykowski \\ Department of Ecology and Silviculture, Faculty of Forestry, University of Agriculture in Kraków, al. 29 Listopada 46, 31-425, Kraków, Poland \\ * Dr. B. Woś, b.wos@ur.krakow.pl , ORCID iD: https://orcid.org/0000-0001-7899-1762
}

Received: 03.06.2020

Accepted: 28.08.2020

Associated editor: Ł. Uzarowicz

\section{Keywords \\ Technosols \\ Industriosols \\ Restoration \\ Carbon stock \\ Soil pH}

\begin{abstract}
This paper presents the diversity of Technosol properties at two post-mining sites: a reclaimed and afforested spoil heap of an opencast sulphur mine and a sandpit excavation, represented by Quaternary sands and loams, Neogene (Krakowiec) clays and mixtures of sands with clays. The investigated post-mining soils significantly differed not only in texture, but also in $\mathrm{pH}$, sorption properties, and $\mathrm{C}$ and $\mathrm{N}$ content. The observed acidification and increase of $\mathrm{C}$ and $\mathrm{N}$ in the uppermost soil layers as compared to the parent material demonstrated how the organic matter that accumulates under the trees influences the soil-forming process. The acidification effect was not so clearly visible on clay soils due to their high buffer capacity. However, soils developed from Neogene clays were characterized by the highest organic carbon stock, which is associated not only with in situ accumulation, but also with geogenic carbon bitumens occurring originally in clays. Less carbon stock was observed in soils formed from mixtures of Quaternary sands and Neogene clays and from Quaternary loams and sands than in Neogene clays. The Technosols indicated progressive pedogenesis initiated by reclamation, which is mainly demonstrated by acidification and increase of $\mathrm{C}$ and $\mathrm{N}$ in the uppermost soil layers horizons compared to the parent rock.
\end{abstract}

\section{Introduction}

The large-scale transformations of the land surface and landscape associated with open-pit mining activities negatively impact the environment. As a result of this exploitation, postmining facilities create above-ground external and internal dumps and deep excavations that radically change water relations both at the facility and in the 'the cone of depression' adjacent to open pit. Open-pit mining devastates the natural soil and plant cover and leaves exposed geological formations in the dumps to become parent rock for future soils (Bell and Donnelly, 2006).

After the occurrence of large-scale ecosystem transformations due to mining, the process of succession begins (Pietrzykowski and Krzaklewski, 2007; Woś et al., 2018). The long-term process of succession in post-mining sites is stimulated by anthropological intervention through reclamation and afforestation (Pietrzykowski and Krzaklewski, 2007). In postmining sites, this process aims to restore the soil function of the newly exposed soil substrates (Rowland et al., 2009). For these reasons, mine-site rehabilitation is presented as an ideal case study for developing an ecosystem starting from point zero (Hüttl and Weber, 2001). Soils on 'natural' sites have evolved over the centuries and their properties are affected and controlled by soil-forming factors like their parent rock, climate, water relations, relief, geographical location, plant cover and land use. Most of them are capable of self-regulation and are characterized by an efficient circulation of nutrients (Bednarek et al., 2011). Developing soils on post-mining sites are characterized by parent material intense transformations that are often derived from a mix of soil substrates from different geological strata, such as Quaternary, Neogene, and Carboniferous strata. These substrates are frequently unfavourable for vegetation and are characterized by properties very rarely found in natural soils: excessive acidification and alkalinity, deficiency of nutrients, low soil organic matter and unsual mineral composition (Pietrzykowski, 2014; Brevik and Lazari, 2014).

Due to the progress of science, the classification of soils formed from post-mining sites is constantly changing. According to Polish Soil Classification (Kabała et al., 2019), soils on postmining sites belong to the anthropogenic soils order, and are called Industriosols. In accordance with the international classification IUSS Working Group WRB (2015), post-mining soils are classified as Spolic Technosols.

This study aimed to compare the properties (texture, $\mathrm{pH}$, nutrient content, sorption complex properties, organic carbon 
stock) of soils formed from Neogene clays and Quaternary sands in the reclaimed spoil heaps of an opencast sulphur mine and a sandpit excavation.

\section{Study area}

The study was carried out at two reclaimed and reforested sites in southern Poland: the spoil heap of a former open-pit sulphur mine Piaseczno (Tarnobrzeg region) and a sand pit mine excavation Szczakowa (Upper Silesia region), south Poland.

The spoil heap of Piaseczno mine is located in the Vistula Lowland (Solon et al., 2018; 50³3.622' N, 21 34.185' E; Fig. 1). In this region, the average annual air temperature is $8.9^{\circ} \mathrm{C}$, and precipitation is $570 \mathrm{~mm}^{\text {year-1 }}{ }^{-1}$ in period 1990-2019 (source: https://en.tutiempo.net). The site is conical in shape with an area of 120 ha and a height of up to $40 \mathrm{~m}$. The spoil heap mainly consists of Neogene clays of the Krakowiec formation, loose Quaternary sands and mixture of Quaternary sands and Neogene clays. During the first 2 years after reclamation carried out in 1970s, initial revegetation and soil stabilization occurred on the spoil heap. During this time, legumes (mainly sweet clover (Melilotus albus L.)) and grasses (e.g., ryegrass (Lolium spp.)) were sown, and mineral fertilization occurred twice with total doses of 80 , 50 and $60 \mathrm{~kg} \mathrm{ha}^{-1}$ of NPK, respectively. In the most difficult areas, where vegetation death occurred due to adverse air-water properties of substrates, sweet clover and alfalfa (Medicago sativa L.) also were sown. Species mainly consisting of oak, pine, birch, larch and alder were planted on the flatter hilltops (Skawina, 1974; Ziemnicki, 1980; Węgorek, 2003).

The Szczakowa sand mine pit is located in the Upper Silesia region, southern Poland (50 $14.394^{\prime} \mathrm{N}, 19^{\circ} 25.140^{\prime} \mathrm{E}$; Fig. 1). In this region, the mean annual air temperature is $8.8^{\circ} \mathrm{C}$, and the precipitation averages $733 \mathrm{~mm}_{\text {year }}{ }^{-1}$ in the period 1990-2019 (source: https://en.tutiempo.net). The open-strip mining resulted in about 3500 ha disturbed area with an excavated depth of 5-25 m. The deposits are genetically related to the fluvioglacial Quaternary sediments deposited in a pre-Quaternary morphological depression. The sedimentation of deposits filling the Biała Przemsza River valley occurred during the Middle Poland and Wörm glaciations period and its approximate age can be defined to reach between 80 and 240 thousand years. In addition to quartz sands, loam layers were found in the deposit. Loam deposits were not removed but stored in the excavation area on the surface. In the studied section of the sand mine cast, reclamation began in the 1970s. Initial reclamation treatments included re-grading the surface and incorporating organic amendments (approximately $300 \mathrm{~m}^{3} \mathrm{ha}^{-1}$ ). The organic amendment was the preserved uppermost forest soil layer of the overlying pre-mining forest (average organic carbon content of $0.3-1.0 \%)$. Subsequent treatments consisted of a two-year fertilization cycle (140, 130 and $150 \mathrm{~kg} \mathrm{ha}^{-1}$ of NPK, respectively) and a two-year cycle of cultivating lupine (Lupinus luteus) and incorporating it into the soil as green manure. The sites were then reforested with Scots pine and a mixture of birch, larch, oak, black locust and alder (Pietrzykowski and Krzaklewski, 2009).

\section{Materials and methods}

A total of 20 plots were selected for the study, taking into account the lithological diversity of the post-mining study objects (Fig. 1). On the Piaseczno spoil heap, the plots were located on 40-year-old post-reclamation soils formed from Quaternary sands (QsP), Neogene clays (Nc) and mixed Quaternary sands and Neogene clays (QsNc). On the Szczakowa excavation, the plots were located on 30-year-old post-reclamation Quaternary sands (QsS) and Quaternary loamy sands and loams (Ql). All plots were established within pure stands of Scots pine (Pinus sylvestris) except for Nc plots, which were located in common oak (Quercus robur) stands.

On each plot, mixed soil samples were taken from the 0-5 cm (A horizon), 5-30 cm and 30-100 cm (C1 and C2 subhorizons). Samples with intact structures were independently collected with $250 \mathrm{~cm}^{3}$ cylinders to determine bulk density (BD). Samples of the organic (litter) horizons (Olf) were collected within five $0.20 \mathrm{~m}^{2}$ square sub-plots. Fresh litter-horizon samples were weighed to the nearest $1 \mathrm{~g}$ with an electronic balance, and mixed samples were considered representative of each plot.

The following properties were determined for mineral horizon: the texture was measured with a Fritsch GmbH Laser Particle Sizer ANALYSETTE 22; pH was measured potentiometrically in $\mathrm{H}_{2} \mathrm{O}$ and $1 \mathrm{M} \mathrm{KCl}$ with a 1:2.5 soil-solution ratio; carbonate $\left(\mathrm{CaCO}_{3}\right)$ content was determined by Scheibler's volumetric method; the organic carbon (SOC) and total nitrogen (Nt) contents were determined using a LECO TruMac ${ }^{\circledR}$ CNS. Prior to carbon analysis, the soil samples were treated with $10 \% \mathrm{HCl}$ to remove carbonates. The content of plant-available phosphorus $\left(\mathrm{P}_{2} \mathrm{O}_{5}\right)$ was determined via the Egner-Riehm method, which used a calcium lactate extract $\left(\left(\mathrm{CH}_{3} \mathrm{CHOHCOO}\right)_{2} \mathrm{Ca}\right)$. The content of exchangeable cations $\left(\mathrm{Ca}^{2+}, \mathrm{K}^{+}, \mathrm{Mg}^{2+}, \mathrm{Na}^{+}\right)$were determined by extraction in $1 \mathrm{M} \mathrm{NH}_{4} \mathrm{Ac}$ and analysis of extract using an ICP-OES iCAP $^{\mathrm{TM}} 6000$ Series spectrophotometer. Total acidity (Hh) was determined via Kappen's method. Cation exchange capacity (CEC) and base saturation (BS) were determined by the sum of exchangeable cations (TEB) and total acidity (Hh).

The organic (litter) horizon samples were oven-dried; the measured moisture loss was used to calculate dry mass. Dried samples were ground, the $\mathrm{C}$ and $\mathrm{N}$ content were determined using a LECO TruMac ${ }^{\circledR}$ CNS and $\mathrm{pH}$ was measured potentiometrically in $1 \mathrm{M} \mathrm{KCl}$ in a 1:5 ratio.

The carbon stocks $\left(\mathrm{C}_{\text {stock }}\right)$ in litter and mineral horizons were calculated using the following equations:

$$
\begin{aligned}
& \mathrm{C}_{\text {stock (itter) }}\left[\mathrm{Mg} \mathrm{ha}^{-1}\right]=\mathrm{M}_{\text {olf }}\left[\mathrm{Mg} \mathrm{ha}^{-1}\right] \times \mathrm{SOC}[\%] / 100 \\
& \mathrm{C}_{\text {stock (mineral) }}\left[\mathrm{Mg} \mathrm{ha}^{-1}\right]=\Sigma\left(\mathrm{SOC}[\%] \times \mathrm{BD}\left[\mathrm{g} \mathrm{cm}^{-3}\right] \times \mathrm{T}[\mathrm{cm}]\right)
\end{aligned}
$$

where: $\mathrm{M}_{\text {olf }}$ is the dry mass of litter horizons; SOC is soil organic carbon content; BD is bulk density; $\mathrm{T}$ is the thickness of the soil horizons.

Statistical analyses were performed using STATISTICA 13.1 software (StatSoft, Inc.). The effects of substrate and depth on 

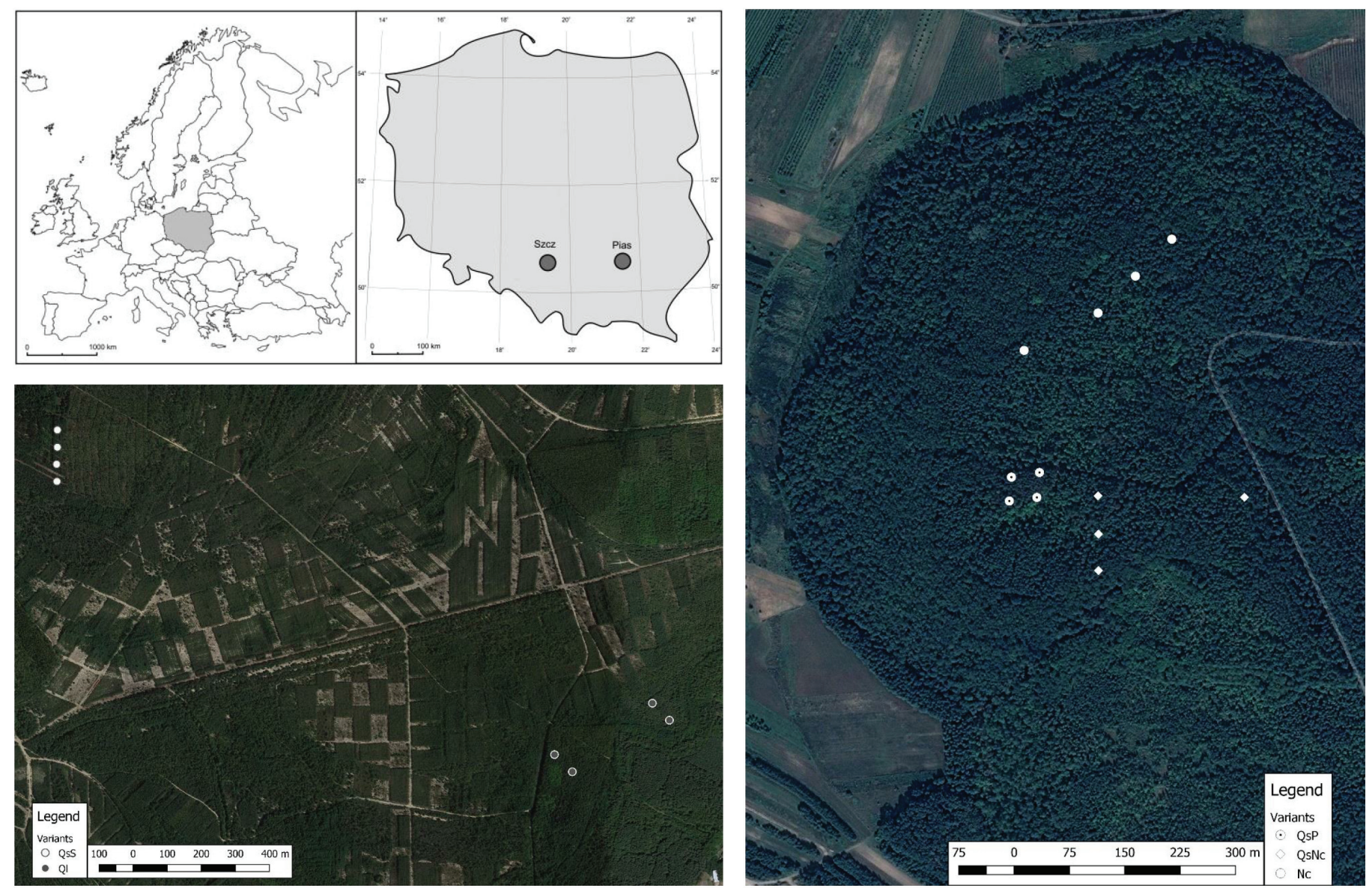

Fig. 1. Location of study sites: a) sand pit mine excavation Szczakowa, and b) spoil heap of sulphur mine Piaseczno

the studied technosol parameters were analysed using two-way ANOVA. The differences (significance assigned at $\mathrm{p}<0.05$ ) between mean values of the measured parameters were calculated using Tukey's test (HSD).

\section{Results}

Soils formed from Quaternary sands (QsP and QsS) were characterized by a sandy texture. Soils formed from mixed sands and clays (QsNc) were classified as loamy sands in the 0-5 and $30-100 \mathrm{~cm}$ horizons and sandy loams texture in the $5-30 \mathrm{~cm}$ horizon. Soils formed from Nc were characterized by a silty loamy texture. The texture of soils formed from Ql in different horizons varied. In $0-5 \mathrm{~cm}$ horizons, these soils were classified as loamy sands, in 5-30 cm sandy loams and in 30-100 cm silt loams. The $\mathrm{BD}$ in the tested soils had the lowest values in the $0-5 \mathrm{~cm}$ horizons (ranging from $1.02 \mathrm{~g} \mathrm{~cm}^{-3}$ on Nc to $1.34 \mathrm{~g} \mathrm{~cm}^{-3}$ on $\mathrm{Ql})$. Carbonates $\left(\mathrm{CaCO}_{3}\right)$ were found in soils formed from $\mathrm{Nc}$ (from 139.5 to $165.1 \mathrm{~g} \mathrm{~kg}^{-1}$ ) and QsNc (ranging from 0.8 to $1.6 \mathrm{~g}$ $\mathrm{kg}^{-1}$; Table 1).

Soil substrate and depth had significant effect on the $\mathrm{pH}$ value, SOC, Nt and $\mathrm{P}_{2} \mathrm{O}_{5}$ content (Table 2; Table 4). The soils formed on the sandpit had lower $\mathrm{pH}$ values compared to the soils formed on the open-cast sulphur mine spoil heap. The $\mathrm{pH}$ in $\mathrm{H}_{2} \mathrm{O}$ of sandpit mineral soil horizons ranged from 4.3 to 5.5, whereas the spoil heap's values ranged from 5.3 to 7.2. The lowest SOC content was found in soils developed from QsS (3.48 $\left.\mathrm{g} \mathrm{kg}^{-1}\right)$, and the highest in soils developed from $\mathrm{Nc}\left(38.80 \mathrm{~g} \mathrm{~kg}^{-1}\right)$. As in the case of SOC, soils formed from QsS displayed the lowest Nt content $\left(0.08 \mathrm{~g} \mathrm{~kg}^{-1}\right.$ in $0-5 \mathrm{~cm}$ horizon). The soils developed from $\mathrm{Nc}$ was characterized by the highest Nt content $\left(3.03 \mathrm{~g} \mathrm{~kg}^{-1}\right)$. The $\mathrm{P}_{2} \mathrm{O}_{5}$ content was very low in soils formed from QsNc, QsS and $\mathrm{Ql}$ (ranging from 0.001 to $0.006 \mathrm{~g} \mathrm{~kg}^{-1}$ ). The higher values were found in the $0-5 \mathrm{~cm}$ horizons of Nc $\left(0.021 \mathrm{~g} \mathrm{~kg}^{-1}\right)$ and QsP $(0.165$ $\mathrm{g} \mathrm{kg}^{-1}$ ) soils (Table 2 ).

The soil substrate had significant effect on the sorption properties (exchangeable cations content, Hh, TEB, CEC and BS). The soil depth and interaction substrate $\times$ soil depth had significant effect on $\mathrm{Hh}, \mathrm{K}^{+}$and BS (Table 3; Table 4). The $0-5 \mathrm{~cm}$ horizons from QsP and Ql soils and all horizons from QsS were characterized by the prevalence of total acidity (Hh) ions in CEC. In other soils and horizons, $\mathrm{Ca}^{2+}$ cations dominated. The content of exchangeable cations in the investigated soils was as follows: $\mathrm{Ca}^{2+}>\mathrm{Mg}^{2+}>\mathrm{K}^{+}>\mathrm{Na}^{+}$. The soil formed from QsS displayed the lowest CEC values. The soils formed from Nc was characterized by the highest CEC values (Table 3).

The lowest $\mathrm{C}_{\text {stock }}$ values were found in soils developed from QsS (15.43 $\mathrm{Mg} \mathrm{ha}^{-1}$ ). The highest $\mathrm{C}_{\text {stock }}$ values were found in soils developed from Nc (168.45 $\mathrm{Mg} \mathrm{ha}^{-1}$; Fig. 2). 
Table 1

Texture, bulk density (BD) and $\mathrm{CaCO}_{3}$ content in the investigated Technosols

\begin{tabular}{|c|c|c|c|c|c|c|c|}
\hline \multirow[t]{2}{*}{ Properties } & & \multirow[t]{2}{*}{ Horizon $[\mathrm{cm}]$} & \multicolumn{5}{|l|}{ Variant } \\
\hline & & & QsP & QsNc & $\mathrm{Nc}$ & QsS & Ql \\
\hline \multirow[t]{3}{*}{ Sand $(2.0-0.05 \mathrm{~mm})$} & {$[\%]$} & $0-5$ & $94 \pm 2^{\mathrm{c} 1}$ & $75 \pm 3^{\mathrm{b}}$ & $6 \pm 1^{\mathrm{a}}$ & $91 \pm 3^{\mathrm{bc}}$ & $84 \pm 7^{\mathrm{bc}}$ \\
\hline & & $5-30$ & $95 \pm 1^{c}$ & $72 \pm 9^{b}$ & $2 \pm 1^{a}$ & $94 \pm 2^{c}$ & $66 \pm 20^{b}$ \\
\hline & & $30-100$ & $96 \pm 0^{\mathrm{d}}$ & $75 \pm 7^{c}$ & $2 \pm 1^{a}$ & $95 \pm 1^{d}$ & $42 \pm 16^{b}$ \\
\hline \multirow[t]{3}{*}{ Silt (0.05-0.002 mm) } & & $0-5$ & $5 \pm 1^{\mathrm{a}}$ & $23 \pm 2^{\mathrm{b}}$ & $78 \pm 2^{\mathrm{c}}$ & $8 \pm 3^{\mathrm{a}}$ & $15 \pm 6^{b}$ \\
\hline & & $5-30$ & $4 \pm 1^{\text {a }}$ & $24 \pm 8^{b}$ & $79 \pm 1^{c}$ & $5 \pm 1^{\mathrm{a}}$ & $31 \pm 18^{\mathrm{b}}$ \\
\hline & & $30-100$ & $3 \pm 0^{\mathrm{a}}$ & $22 \pm 6^{b}$ & $77 \pm 1^{d}$ & $4 \pm 1^{\mathrm{a}}$ & $52 \pm 14^{c}$ \\
\hline \multirow[t]{3}{*}{ Clay $(<0.002 \mathrm{~mm})$} & & $0-5$ & $1 \pm 0^{\mathrm{a}}$ & $3 \pm 0^{\mathrm{a}}$ & $16 \pm 2^{\mathrm{b}}$ & $1 \pm 0^{\mathrm{a}}$ & $2 \pm 1^{\mathrm{a}}$ \\
\hline & & $5-30$ & $1 \pm 0^{a}$ & $4 \pm 2^{\mathrm{b}}$ & $19 \pm 1^{c}$ & $1 \pm 0^{\mathrm{a}}$ & $4 \pm 2^{\mathrm{b}}$ \\
\hline & & $30-100$ & $1 \pm 0^{\mathrm{a}}$ & $3 \pm 1^{b}$ & $21 \pm 1^{\mathrm{d}}$ & $1 \pm 0^{\mathrm{a}}$ & $6 \pm 2^{c}$ \\
\hline \multirow[t]{3}{*}{$\mathrm{BD}$} & {$\left[\mathrm{g} \mathrm{cm}^{3}\right]$} & $0-5$ & $1.41 \pm 0.08^{b}$ & $1.34 \pm 0.05^{b}$ & $1.02 \pm 0.09^{a}$ & $1.38 \pm 0.06^{\mathrm{b}}$ & $1.43 \pm 0.07^{b}$ \\
\hline & & $5-30$ & $1.58 \pm 0.07^{b}$ & $1.49 \pm 0.06^{b}$ & $1.24 \pm 0.10^{\mathrm{a}}$ & $1.52 \pm 0.03^{\mathrm{b}}$ & $1.67 \pm 0.08^{b}$ \\
\hline & & $30-100$ & $1.62 \pm 0.05^{b}$ & $1.61 \pm 0.04^{b}$ & $1.23 \pm 0.03^{\mathrm{a}}$ & $1.61 \pm 0.06^{\mathrm{b}}$ & $1.63 \pm 0.09^{b}$ \\
\hline \multirow[t]{3}{*}{$\mathrm{CaCO}_{3}$} & {$\left[\mathrm{~g} \mathrm{~kg}^{-1}\right]$} & $0-5$ & n.f. ${ }^{2}$ & $0.8 \pm 0.7^{\mathrm{a}}$ & $165.1 \pm 17.2^{\mathrm{b}}$ & n.f. & n.f. \\
\hline & & $5-30$ & n.f. & $1.6 \pm 2.4^{\mathrm{a}}$ & $163.0 \pm 25.8^{b}$ & n.f. & n.f. \\
\hline & & $30-100$ & n.f. & $1.3 \pm 1.4^{\mathrm{a}}$ & $139.5 \pm 68.8^{\mathrm{b}}$ & n.f. & n.f. \\
\hline
\end{tabular}

${ }^{1}$ mean \pm SD, different letters $(a, b, c)$ indicate significant $(p<0.05)$ differences between the studied variants

${ }^{2}$ n.f. - not found.

Table 2

Reaction, carbon, nitrogen and available phosphorus content in the investigated soils

\begin{tabular}{|c|c|c|c|c|c|c|c|}
\hline \multirow{2}{*}{\multicolumn{2}{|c|}{ Properties }} & \multirow[t]{2}{*}{ Horizon $[\mathrm{cm}]$} & \multicolumn{5}{|l|}{ Variant } \\
\hline & & & QsP & QsNc & $\mathrm{Nc}$ & QsS & Ql \\
\hline \multirow{4}{*}{\multicolumn{2}{|c|}{$\mathrm{pH}$ in $\mathrm{H}_{2} \mathrm{O}$}} & Olf & $4.4 \pm 0.2^{\text {bc } 1}$ & $4.8 \pm 0.3^{c}$ & $5.9 \pm 0.2^{\mathrm{d}}$ & $3.9 \pm 0.1^{\mathrm{a}}$ & $4.1 \pm 0.2^{\mathrm{ab}}$ \\
\hline & & $0-5$ & $5.3 \pm 0.1^{\mathrm{ab}}$ & $5.7 \pm 0.7^{b}$ & $7.1 \pm 0.1^{\mathrm{c}}$ & $4.7 \pm 0.1^{\mathrm{a}}$ & $4.3 \pm 0.1^{\mathrm{a}}$ \\
\hline & & $5-30$ & $6.3 \pm 0.9^{b}$ & $6.4 \pm 0.6^{\mathrm{b}}$ & $7.0 \pm 0.2^{\mathrm{b}}$ & $5.1 \pm 0.1^{\mathrm{a}}$ & $4.9 \pm 0.4^{\mathrm{a}}$ \\
\hline & & $30-100$ & $7.0 \pm 0.6^{b}$ & $6.6 \pm 0.9^{b}$ & $7.2 \pm 0.2^{\mathrm{b}}$ & $5.5 \pm 0.1^{\mathrm{a}}$ & $5.3 \pm 0.4^{\mathrm{a}}$ \\
\hline \multirow{4}{*}{\multicolumn{2}{|c|}{$\mathrm{pH}$ in $\mathrm{KCl}$}} & Olf & $3.7 \pm 0.2^{\mathrm{bc}}$ & $4.2 \pm 0.3^{c}$ & $5.6 \pm 0.4^{\mathrm{d}}$ & $3.2 \pm 0.1^{\mathrm{a}}$ & $3.6 \pm 0.2^{\mathrm{ab}}$ \\
\hline & & $0-5$ & $4.4 \pm 0.1^{\mathrm{a}}$ & $5.0 \pm 0.9^{b}$ & $6.7 \pm 0.0^{c}$ & $3.9 \pm 0.2^{\mathrm{a}}$ & $3.7 \pm 0.2^{\mathrm{a}}$ \\
\hline & & $5-30$ & $5.7 \pm 0.8^{\mathrm{b}}$ & $5.6 \pm 0.9^{b}$ & $6.6 \pm 0.2^{\mathrm{b}}$ & $4.1 \pm 0.1^{\mathrm{a}}$ & $3.9 \pm 0.4^{\mathrm{a}}$ \\
\hline & & $30-100$ & $6.4 \pm 0.6^{\mathrm{b}}$ & $5.8 \pm 1.2^{\mathrm{b}}$ & $6.8 \pm 0.1^{\mathrm{b}}$ & $4.4 \pm 0.1^{\mathrm{a}}$ & $4.2 \pm 0.4^{\mathrm{a}}$ \\
\hline \multirow{4}{*}{\multicolumn{2}{|c|}{$\left[\mathrm{g} \mathrm{kg}^{-1}\right]$}} & Olf & $421.95 \pm 29.21^{\mathrm{a}}$ & $380.65 \pm 35.25^{a}$ & $403.95 \pm 28.75^{\mathrm{a}}$ & $421.29 \pm 19.82^{\mathrm{a}}$ & $417.33 \pm 12.60^{\mathrm{a}}$ \\
\hline & & $0-5$ & $7.56 \pm 4.21^{\mathrm{a}}$ & $18.39 \pm 3.56^{\mathrm{b}}$ & $38.80 \pm 4.70^{c}$ & $3.48 \pm 0.79^{\mathrm{a}}$ & $7.58 \pm 2.52^{\mathrm{a}}$ \\
\hline & & $5-30$ & $0.99 \pm 0.32^{\mathrm{a}}$ & $3.10 \pm 0.98^{b}$ & $12.59 \pm 5.02^{c}$ & $0.81 \pm 0.19^{\mathrm{a}}$ & $1.53 \pm 0.17^{\mathrm{a}}$ \\
\hline & & $30-100$ & $0.40 \pm 0.11^{\mathrm{a}}$ & $1.41 \pm 1.26^{\mathrm{b}}$ & $12.20 \pm 1.78^{c}$ & $0.13 \pm 0.03^{\mathrm{a}}$ & $0.40 \pm 0.11^{\mathrm{a}}$ \\
\hline \multirow{4}{*}{\multicolumn{2}{|c|}{$\mathrm{Nt}$}} & Olf & $11.63 \pm 0.91^{\mathrm{a}}$ & $11.62 \pm 1.52^{\mathrm{a}}$ & $18.33 \pm 1.41^{\mathrm{a}}$ & $10.12 \pm 0.84^{\mathrm{a}}$ & $11.25 \pm 1.38^{\mathrm{a}}$ \\
\hline & & $0-5$ & $0.29 \pm 0.18^{\mathrm{a}}$ & $1.02 \pm 0.20^{\mathrm{b}}$ & $3.03 \pm 0.49^{c}$ & $0.08 \pm 0.02^{\mathrm{a}}$ & $0.24 \pm 0.08^{a}$ \\
\hline & & $5-30$ & $0.01 \pm 0.01^{\mathrm{a}}$ & $0.17 \pm 0.05^{b}$ & $0.84 \pm 0.31^{c}$ & $0.01 \pm 0.00^{\mathrm{a}}$ & $0.03 \pm 0.03^{a}$ \\
\hline & & $30-100$ & n.f. ${ }^{2}$ & $0.09 \pm 0.10^{\mathrm{a}}$ & $0.55 \pm 0.11^{\mathrm{b}}$ & n.f. & $0.02 \pm 0.01^{\mathrm{a}}$ \\
\hline \multirow{3}{*}{\multicolumn{2}{|c|}{$\mathrm{P}_{2} \mathrm{O}_{5}$}} & $0-5$ & $0.165 \pm 0.109^{b}$ & $0.006 \pm 0.003^{a}$ & $0.021 \pm 0.003^{a}$ & $0.001 \pm 0.001^{\mathrm{a}}$ & $0.002 \pm 0.002^{\mathrm{a}}$ \\
\hline & & $5-30$ & $0.007 \pm 0.014^{\mathrm{a}}$ & $0.006 \pm 0.004^{\mathrm{a}}$ & $0.012 \pm 0.001^{\mathrm{a}}$ & $0.001 \pm 0.000^{\mathrm{a}}$ & $0.001 \pm 0.000^{\mathrm{a}}$ \\
\hline & & $30-100$ & n.f. & $0.004 \pm 0.004^{\mathrm{a}}$ & $0.011 \pm 0.003^{\mathrm{a}}$ & $0.001 \pm 0.000^{\mathrm{a}}$ & $0.001 \pm 0.000^{\mathrm{a}}$ \\
\hline
\end{tabular}

${ }^{1}$ mean \pm SD, different letters $(a, b, c)$ indicate significant $(\mathrm{p}<0.05)$ differences between studied variants.

${ }^{2}$ n.f. - not found. 
Table 3

Sorption complex properties in the investigated post-mining soils

\begin{tabular}{|c|c|c|c|c|c|c|c|}
\hline \multirow[t]{2}{*}{ Properties } & & \multirow[t]{2}{*}{ Horizon $[\mathrm{cm}]$} & \multicolumn{5}{|l|}{ Variant } \\
\hline & & & QsP & QsNc & $\mathrm{Nc}$ & QsS & Ql \\
\hline \multirow[t]{3}{*}{$\mathrm{Hh}$} & {$\left[\mathrm{cmol}(+) \mathrm{kg}^{-1}\right]$} & $0-5$ & $2.06 \pm 0.18^{\mathrm{a} 1}$ & $3.70 \pm 1.23^{b}$ & $1.21 \pm 0.06^{\mathrm{a}}$ & $1.96 \pm 0.23^{\mathrm{a}}$ & $3.53 \pm 0.71^{\mathrm{b}}$ \\
\hline & & $5-30$ & $0.67 \pm 0.14^{\mathrm{a}}$ & $1.12 \pm 0.23^{\mathrm{a}}$ & $0.90 \pm 0.06^{\mathrm{a}}$ & $0.96 \pm 0.13^{\mathrm{a}}$ & $1.55 \pm 0.38^{\mathrm{a}}$ \\
\hline & & $30-100$ & $0.55 \pm 0.17^{\mathrm{a}}$ & $0.86 \pm 0.25^{\mathrm{a}}$ & $0.80 \pm 0.08^{\mathrm{a}}$ & $0.70 \pm 0.03^{\mathrm{a}}$ & $1.35 \pm 0.33^{\mathrm{a}}$ \\
\hline \multirow[t]{3}{*}{$\mathrm{Ca}^{2+}$} & & $0-5$ & $1.34 \pm 0.56^{\mathrm{a}}$ & $5.71 \pm 1.59^{a}$ & $42.31 \pm 2.95^{\mathrm{b}}$ & $0.39 \pm 0.19^{a}$ & $0.63 \pm 0.39^{a}$ \\
\hline & & $5-30$ & $0.90 \pm 0.42^{\mathrm{a}}$ & $5.30 \pm 4.15^{\mathrm{a}}$ & $48.58 \pm 9.08^{b}$ & $0.42 \pm 0.08^{a}$ & $1.66 \pm 0.82^{\mathrm{a}}$ \\
\hline & & $30-100$ & $1.89 \pm 2.44^{\mathrm{a}}$ & $5.65 \pm 4.02^{\mathrm{a}}$ & $60.99 \pm 19.89^{b}$ & $0.40 \pm 0.02^{\mathrm{a}}$ & $3.72 \pm 0.94^{\mathrm{a}}$ \\
\hline \multirow[t]{3}{*}{$\mathrm{K}^{+}$} & & $0-5$ & $0.05 \pm 0.01^{\mathrm{a}}$ & $0.13 \pm 0.04^{\mathrm{a}}$ & $0.92 \pm 0.10^{\mathrm{b}}$ & $0.05 \pm 0.01^{\mathrm{a}}$ & $0.07 \pm 0.02^{\mathrm{a}}$ \\
\hline & & $5-30$ & $0.03 \pm 0.01^{\mathrm{a}}$ & $0.05 \pm 0.02^{\mathrm{a}}$ & $0.61 \pm 0.10^{\mathrm{b}}$ & $0.03 \pm 0.01^{\mathrm{a}}$ & $0.10 \pm 0.03^{\mathrm{a}}$ \\
\hline & & $30-100$ & $0.02 \pm 0.01^{\mathrm{a}}$ & $0.05 \pm 0.03^{\mathrm{a}}$ & $0.68 \pm 0.30^{\mathrm{b}}$ & $0.03 \pm 0.01^{\mathrm{a}}$ & $0.14 \pm 0.03^{\mathrm{a}}$ \\
\hline \multirow[t]{3}{*}{$\mathrm{Mg}^{2+}$} & & $0-5$ & $0.13 \pm 0.06^{a}$ & $0.54 \pm 0.01^{b}$ & $1.18 \pm 0.21^{c}$ & $0.11 \pm 0.05^{a}$ & $0.14 \pm 0.09^{a}$ \\
\hline & & $5-30$ & $0.01 \pm 0.01^{\mathrm{a}}$ & $0.29 \pm 0.13^{\mathrm{ab}}$ & $0.68 \pm 0.52^{c}$ & $0.15 \pm 0.04^{\mathrm{a}}$ & $0.53 \pm 0.25^{b c}$ \\
\hline & & $30-100$ & $0.01 \pm 0.00^{\mathrm{a}}$ & $0.35 \pm 0.24^{\mathrm{ab}}$ & $1.17 \pm 0.78^{c}$ & $0.18 \pm 0.03^{\mathrm{a}}$ & $0.87 \pm 0.20^{\mathrm{bc}}$ \\
\hline \multirow[t]{3}{*}{$\mathrm{Na}^{+}$} & & $0-5$ & $0.01 \pm 0.01^{\mathrm{a}}$ & $0.02 \pm 0.00^{\mathrm{a}}$ & $0.07 \pm 0.01^{\mathrm{b}}$ & $0.01 \pm 0.00^{\mathrm{a}}$ & $0.02 \pm 0.00^{\mathrm{a}}$ \\
\hline & & $5-30$ & $0.01 \pm 0.01^{\mathrm{a}}$ & $0.02 \pm 0.01^{\mathrm{a}}$ & $0.07 \pm 0.01^{\mathrm{b}}$ & $0.01 \pm 0.00^{\mathrm{a}}$ & $0.02 \pm 0.01^{\mathrm{a}}$ \\
\hline & & $30-100$ & $0.01 \pm 0.01^{\mathrm{a}}$ & $0.02 \pm 0.01^{\mathrm{a}}$ & $0.06 \pm 0.00^{\mathrm{a}}$ & $0.01 \pm 0.00^{\mathrm{a}}$ & $0.02 \pm 0.00^{\mathrm{a}}$ \\
\hline \multirow[t]{3}{*}{ TEB } & & $0-5$ & $1.53 \pm 0.63^{\mathrm{a}}$ & $6.40 \pm 1.61^{\mathrm{a}}$ & $44.49 \pm 3.11^{\mathrm{b}}$ & $0.56 \pm 0.25^{\mathrm{a}}$ & $0.87 \pm 0.50^{\mathrm{a}}$ \\
\hline & & $5-30$ & $0.96 \pm 0.42^{\mathrm{a}}$ & $5.66 \pm 4.25^{\mathrm{a}}$ & $49.93 \pm 9.49^{b}$ & $0.62 \pm 0.12^{\mathrm{a}}$ & $2.31 \pm 1.10^{\mathrm{a}}$ \\
\hline & & $30-100$ & $1.92 \pm 2.44^{\mathrm{a}}$ & $6.07 \pm 4.23^{\mathrm{a}}$ & $62.91 \pm 20.77^{b}$ & $0.61 \pm 0.05^{a}$ & $4.76 \pm 1.16^{\mathrm{a}}$ \\
\hline \multirow[t]{3}{*}{ CEC } & & $0-5$ & $3.59 \pm 1.10^{\mathrm{a}}$ & $10.11 \pm 0.75^{b}$ & $45.70 \pm 3.16^{c}$ & $2.53 \pm 0.42^{\mathrm{a}}$ & $4.39 \pm 1.13^{a}$ \\
\hline & & $5-30$ & $1.63 \pm 0.28^{\mathrm{a}}$ & $6.78 \pm 4.01^{\mathrm{a}}$ & $50.83 \pm 9.46^{b}$ & $1.58 \pm 0.21^{\mathrm{a}}$ & $3.86 \pm 1.43^{a}$ \\
\hline & & $30-100$ & $2.47 \pm 2.44^{\mathrm{a}}$ & $6.93 \pm 4.11^{\mathrm{a}}$ & $63.71 \pm 20.81^{b}$ & $1.32 \pm 0.05^{\mathrm{a}}$ & $6.12 \pm 1.04^{a}$ \\
\hline \multirow[t]{3}{*}{ BS } & {$[\%]$} & $0-5$ & $41.74 \pm 6.82^{\mathrm{a}}$ & $63.03 \pm 12.84^{\mathrm{b}}$ & $97.36 \pm 0.08^{c}$ & $21.6 \pm 7.44^{\mathrm{a}}$ & $18.66 \pm 7.02^{\mathrm{a}}$ \\
\hline & & $5-30$ & $56.80 \pm 16.35^{a}$ & $78.91 \pm 10.45^{b}$ & $98.16 \pm 0.46^{c}$ & $39.1 \pm 4.04^{\mathrm{a}}$ & $57.81 \pm 9.67^{\mathrm{a}}$ \\
\hline & & $30-100$ & $63.84 \pm 24.69^{a b}$ & $82.35 \pm 14.76^{b}$ & $98.61 \pm 0.56^{c}$ & $46.5 \pm 2.66^{\mathrm{a}}$ & $77.32 \pm 7.28^{b}$ \\
\hline
\end{tabular}

${ }^{1}$ mean \pm SD, different letters $(a, b, c)$ indicate significant differences between the variants.

Fig. 2. Carbon stocks $\left(\mathrm{C}_{\text {stock }}\right)$ in investigated post-mining soils. Means followed by different letters (a, b, c) are significantly different

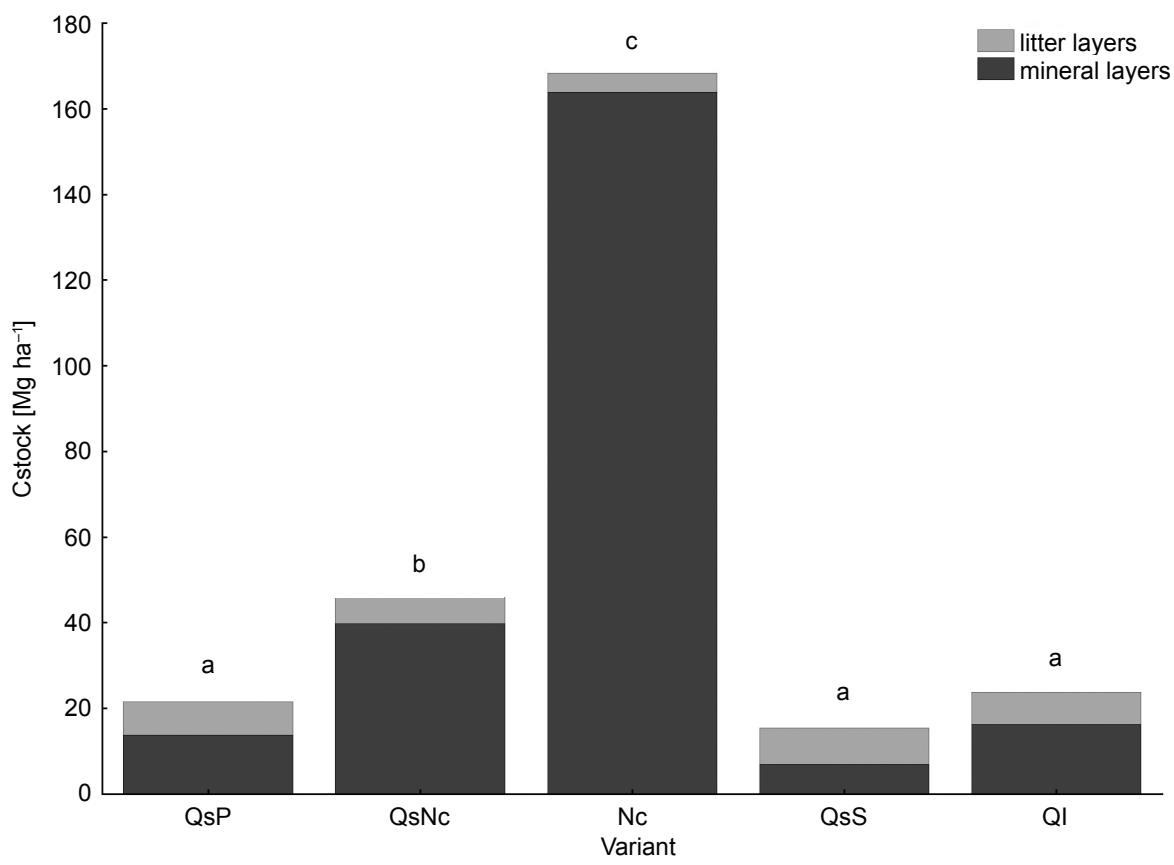


Table 4

Two-way ANOVA results for properties of Technosols on investigated post-mining sites

\begin{tabular}{|c|c|c|c|c|c|c|}
\hline \multirow[t]{2}{*}{ Properties } & \multicolumn{2}{|c|}{ Substrate } & \multicolumn{2}{|c|}{ Soil depth } & \multicolumn{2}{|c|}{ Interaction Substrate $\times$ Soil depth } \\
\hline & $\mathrm{F}$ & $\mathrm{p}$ & $\mathrm{F}$ & $\mathrm{p}$ & $\mathrm{F}$ & $\mathrm{p}$ \\
\hline Sand & 289.00 & 0.0000 & 5.76 & 0.0059 & 6.68 & 0.0000 \\
\hline Silt & 233.33 & 0.0000 & 4.08 & 0.0235 & 6.83 & 0.0000 \\
\hline Clay & 551.71 & 0.0000 & 16.08 & 0.0000 & 5.09 & 0.0002 \\
\hline $\mathrm{CaCO}_{3}$ & 152.11 & 0.0000 & n.s. ${ }^{1}$ & n.s. & n.s. & n.s. \\
\hline $\mathrm{pH}$ in $\mathrm{H}_{2} \mathrm{O}$ & 49.89 & 0.0000 & 20.50 & 0.0000 & n.s. & n.s. \\
\hline $\mathrm{pH}$ in $\mathrm{KCl}$ & 51.57 & 0.0000 & 10.43 & 0.0002 & n.s. & n.s. \\
\hline SOC & 130.58 & 0.0000 & 154.60 & 0.0000 & 19.95 & 0.0000 \\
\hline Nt & 150.45 & 0.0000 & 132.22 & 0.0000 & 40.26 & 0.0000 \\
\hline $\mathrm{P}_{2} \mathrm{O}_{5}$ & 7.72 & 0.0001 & 9.42 & 0.0004 & 7.70 & 0.0000 \\
\hline $\mathrm{Hh}$ & 17.49 & 0.0000 & 85.58 & 0.0000 & 5.78 & 0.0001 \\
\hline $\mathrm{Ca}^{2+}$ & 159.40 & 0.0000 & n.s. & n.s. & n.s. & n.s. \\
\hline $\mathrm{K}^{+}$ & 145.83 & 0.0000 & 4.74 & 0.0136 & 2.93 & 0.0102 \\
\hline $\mathrm{Mg}^{2+}$ & 23.06 & 0.0000 & n.s. & n.s. & n.s. & n.s. \\
\hline $\mathrm{Na}^{+}$ & 192.52 & 0.0000 & n.s. & n.s. & n.s. & n.s. \\
\hline TEB & 155.30 & 0.0000 & n.s. & n.s. & n.s. & n.s. \\
\hline CEC & 152.11 & 0.0000 & n.s. & n.s. & n.s. & n.s. \\
\hline BS & 61.93 & 0.0000 & 29.80 & 0.0000 & 4.00 & 0.0012 \\
\hline
\end{tabular}

${ }^{1}$ n.s. - not significant $(\mathrm{p}<0.05)$.

\section{Discussion}

One of the most important stages of soil-forming process in post-mining sites is the accumulation of organic matter in the uppermost soil horizons (Pietrzykowski and Krzaklewski, 2007; Brevik and Lazari, 2014.). In the investigated soils, the SOC increase in the $0-5 \mathrm{~cm}$ horizons (ranging from $3.35 \mathrm{~g} \mathrm{~kg}^{-1}$ in PczS to $26.60 \mathrm{~g} \mathrm{~kg}^{-1}$ in Nc) as compared to the parent rock (30-100 cm). The $\mathrm{C}_{\text {stock }}$ in QsP, QsNc, QsS and Q1 had lower values, and in the case of Nc higher values, than previously reported for forest soils in 'natural' sites. Data from the National Forest Soil Inventory (NFSI) indicate that the $\mathrm{C}_{\text {stock }}$ in soil horizons (up to $100 \mathrm{~cm}$ deep) at locations throughout Poland is, on average, $26 \mathrm{Mg} \mathrm{ha}^{-1}$ in the organic horizons and $66 \mathrm{Mg} \mathrm{ha}^{-1}$ in the mineral horizons (Gruba and Socha, 2019). These values were similar in the organic horizons and lower in the mineral horizons (up to 1 meter deep) than reported in applicable European forests literature (22.1 $\mathrm{Mg} \mathrm{C} \mathrm{ha}^{-1}$ and $108 \mathrm{Mg} \mathrm{C} \mathrm{ha}^{-1}$, respectively) (De Vos et al., 2015), which the authors explain is due to the propensity of pine stands to grow on poor sandy soils in Poland (Gruba and Socha, 2019). However, the study's results are consistent with the ranges observed in other post-mining sites. Vindušková and Frouz (2013) reported that the $C_{\text {stock }}$ in post-mining soils in the northern hemisphere (Europe and the USA) can range from 4.49 to $93.20 \mathrm{Mg} \mathrm{ha}^{-1}$. Pietrzykowski and Daniels (2014) found that the $\mathrm{C}_{\text {stock }}$ (up to $110 \mathrm{~cm}$ ) in soils at various post-mining sites in Poland ranges from $16.8 \mathrm{Mg} \mathrm{ha}^{-1}$ in the Quaternary sands of the Szczakowa mine to $2975.2 \mathrm{Mg} \mathrm{ha}^{-1}$ in the carbonaceous shales of the Smolnica overburden spoil heap. However, such an extremely high $\mathrm{C}_{\text {stock }}$ resulted from the presence of hard coal on the Smolnica heap.
The higher values noted in soils developed from QsNc and Nc confirmed the fact that soils with a higher silt and clay content are typically characterized by a higher content of organic carbon. This difference is due to SOC binding by clay particles in the form of organic-mineral complexes. These complexes are resistant to leaching and microbiological degradation (Laird et al., 2001; Jimenez and Lal, 2006). The highest C content, observed in QsNc and Nc soils, was also due to the fossil (geogenic) carbon that accumulated in previous geological epochs during clay deposition (Pawłowski et al., 1985). The fossil carbon affects the overall soil carbon balance in initial ecosystems; it is also important for soil water retention (Fettweis et al., 2005; Frouz, 2016). However, the process of its incorporation into the biogeochemical cycle has not been thoroughly studied, although it is known that soil microorganisms play a key role in the process (Rumpel and Kögel-Knabner, 2003; Fettweis et al., 2005; Frouz, 2016). Determining the amount of fossil carbon in the total organic carbon pool in post-mining soils is difficult from a methodological point of view (Chabbi et al., 2008). The content of fossil carbon was determined based on the degree of $14 \mathrm{C}$ radioactive isotope decay (Rumpel and Kögel-Knabner, 2003) and via formulas that take into account specific post-mining site indicators (Amichev et al., 2008). Due to the young age of the studied soils and relatively poor accumulation of organic matter from decomposition in situ, the content of geogenic carbon can be approximated by the C content in the parent rock horizons (Vindušková and Frouz, 2013). This value averaged $1.41 \mathrm{~g} \mathrm{~kg}^{-1}$ for QsNc and $12.20 \mathrm{~g} \mathrm{~kg}^{-1}$ for Nc clays. Gruszczyński et al. (2012) reported slightly lower values in the nearby Machów mine, indicating that the Neogene (Krakowiec) clays have an organic carbon content ranging from 6.0 to $10.0 \mathrm{~g} \mathrm{~kg}^{-1}$. 
Dynamic of pedogenesis is also indicated by an increase in the Nt content (ranging from $0.08 \mathrm{~g} \mathrm{~kg}^{-1}$ on QsS to $2.48 \mathrm{~g} \mathrm{~kg}^{-1}$ on $\mathrm{Nc}$ ) in $0-5 \mathrm{~cm}$ horizons as compared to the parent rock. As in the case of SOC, the higher Nt content in soils formed from QsNc and Nc is partly due to the genesis of the Neogene clays, and not as a result of accumulation in situ (Pietrzykowski et al., 2010). The content of fossil $\mathrm{N}$ accumulated in clays can be approximated in the parent rock horizons at $0.09 \mathrm{~g} \mathrm{~kg}^{-1}$ in QsNc and $0.55 \mathrm{~g} \mathrm{~kg}^{-1}$ in Nc. There is conflicting information regarding the fossil $\mathrm{N}$ availability for soil microorganisms and its incorporation into the biological cycle. Studies carried out at the hard coal mining spoil heaps indicate that the $\mathrm{N}$ contained therein is a heterocyclic compound and is therefore unavailable to plants and not included in biological circulation (Strzyszcz and Harabin, 2004). Research performed at the post-mining sites after lignite exploitation indicates that $\mathrm{N}$ availability from lignite was low in young stands and higher in older stands (Hüttl and Weber, 2001).

One of the most frequently observed processes in the early stages of the pedogenesis on reclaimed post-mining sites is a decrease in the $\mathrm{pH}$ value in uppermost soil horizons (Pietrzykowski and Krzaklewski, 2009). The decrease in $\mathrm{pH}$ measured in $\mathrm{H}_{2} \mathrm{O}$ from the $0-5 \mathrm{~cm}$ horizons as compared to parent rock ranged from 0.12 in Nc to 1.75 in QsP, whereas the difference in $\mathrm{KCl}$ ranged from 0.07 in Nc to 2.07 in QsP. The lack of a significant decrease in the $\mathrm{pH}$ of soils formed from Neogene clays was due to this substrate's high buffer capacity and carbonates content (Józefowska et al., 2019).

The quantitative series of exchangeable cations in the studied soils $\left(\mathrm{Ca}^{2+}>\mathrm{Mg}^{2+}>\mathrm{K}^{+}>\mathrm{Na}^{+}\right)$was similar to natural soils occurring in the temperate climate zone (Bednarek et al., 2011). As with the decrease in $\mathrm{pH}$ value, the increase in total acidity (Hh) in uppermost soil horizons as compared to the parent rock levels is an indicator of pedogenesis.

The available phosphorus content in the studied soils was low, indicating a deficit of this element (Baule and Fricker, 1973). Phosphorus deficiency in soils has been reported in other post-mining sites reclaimed for forestry (Pietrzykowski et al., 2010).

\section{Conclusions}

The obtained results permitted drawing the following conclusions:

- The investigated technogenic soils significantly differed not only in texture, but also in $\mathrm{pH}$, sorption properties, $\mathrm{C}$ and $\mathrm{N}$ content.

- The soils formed from Neogene clays were characterized by the highest carbon stock, which is associated not only with in situ accumulation of soil organic matter, but also with geogenic carbon bitumens occurring originally in clays.

- The technogenic soils properties indicated progressive pedogenesis which is demonstrated by acidification and increase of $\mathrm{C}$ and $\mathrm{N}$ content in the uppermost soil horizons compared to the parent rock.

\section{Acknowledgments}

Article was financed by a subvention from the Ministry of Science and Higher Education of the Republic of Poland for the University of Agriculture in Krakow for 2020.

\section{References}

Amichev, B.Y., Burger, J.A., Rodrigue, J.A., 2008. Carbon sequestration by forests and soils on mined land in the Midwestern and Appalachian coalfields of the U.S. Forest Ecology and Management 256, 1949-1959. https://doi.org/10.1016/j.foreco.2008.07.020

Baule, H., Fricker, C., 1973. Nawożenie drzew leśnych. PWRiL, Warszawa.

Bednarek, R., Dziadowiec, H., Pokojska, U., Prusinkiewicz, Z., 2011. Badania ekologiczno-gleboznawcze. Wydawnictwo Naukowe PWN, Warszawa.

Bell, F.G., Donnelly, L.J., 2006. Mining and its impact on the environment. Taylor and Francis Group, London and New York.

Brevik, E.C., Lazari, A.G., 2014. Rates of Pedogenesis in Reclaimed Lands as Compared to Rates of Natural Pedogenesis. Soil Horizons 55(1), 1-6. https://doi.org/10.2136/sh13-06-0017

Chabbi, A., Sebilo, M., Rumpel, C., Schaaf, W., Mariotti, A., 2008. Origin of nitrogen in reforested lignite-rich mine soils revealed by stable isotope analysis. Environmental Science \& Technology 42, 2787-2792. https://doi.org/10.1021/es702377k

de Vos, B., Cools, N., Ilvesniemi, H., Vesterdal, L., Vanguelova, E., Carnicelli, S., 2015. Benchmark values for forest soil carbon stocks in Europe: Results from a large scale forest soil survey. Geoderma 251-252, 33-46. https://doi.org/10.1016/j.geoderma.2015.03.008

Fettweis, U., Bens, O., Hüttl, R.F., 2005. Accumulation and properties of soil organic carbon at reclaimed sites in the Lusatian lignite mining district afforested with Pinus sp. Geoderma 129(1-2), 81-91. https:// doi.org/10.1016/j.geoderma.2004.12.034

Frouz, J., 2016. Effects of Soil Development Time and Litter Quality on Soil Carbon Sequestration: Assessing Soil Carbon Saturation with a Field Transplant Experiment along a Post-mining Chronosequence. Land Degradation \& Development 28(2), 664-672. https://doi.org/10.1002/ ldr. 2580

Gruba, P., Socha, J., 2019. Exploring the effects of dominant forest tree species, soil texture, altitude, and $\mathrm{pH}_{\mathrm{H} 2 \mathrm{O}}$ on soil carbon stocks using generalized additive models. Forest Ecology and Management 447, 105-114. https://doi.org/10.1016/j.foreco.2019.05.061

Gruszczyński, S., Sroka, K., Trafas, M., 2012. Akumulacja węgla organicznego w iłach krakowieckich zrekultywowanego zwałowiska kopalni siarki „Machów”. Przegląd Górniczy 68(8), 27-36.

Hüttl, R.F., Weber, E., 2001. Forest ecosystem development in post-mining landscapes: a case study of the Lusatian lignite district. Naturwissenschaften 88, 322-329. https://doi.org/10.1007/s001140100241

IUSS Working Group WRB, 2015. World Reference Base for soil resources 2014. International soil classification system for naming soils and creating legends for soil maps. Update 2015. World Soil Resources Report No. 106. FAO, Rome: 212 pp.

Józefowska, A., Sokołowska, J., Woźnica, K., Woś, B., Pietrzykowski, M., 2019. Tree species and soil substrate affect buffer capacity of anthroposols in afforested postmine sites in Poland. Journal of Soil and Water Conservation 74(4), 372-379. https://doi.org/10.2489/jswc.74.4.372

Kabała C., Charzyński P., Chodorowski J., Drewnik M., Glina B., Greinert A., Hulisz P., Jankowski M., Jonczak J., Łabaz B., Łachacz A., Marzec M., Mendyk Ł., Musiał P., Musielok Ł., Smreczak B., Sowiński P., Świtoniak M., Uzarowicz Ł., Waroszewski J., 2019. Polish Soil Classification, 6th edition - principles, classification scheme and correlations. Soil Science Annual 70(2), 71-97. https://doi.org/10.2478/ssa2019-0009 
Pawłowski S., Pawłowska K., Kubica B., 1985. Budowa geologiczna tarnobrzeskiego złoża siarki rodzimej. Red. K. Pawłowska. Prace Instytutu Geologicznego CXIV, Wydawnictwa Geologiczne, Warszawa.

Pietrzykowski, M., Krzaklewski, W., Pająk, M., Socha, J., Ochał, W., 2010. Analiza i optymalizacja metod klasyfikacji siedlisk i kryteriów oceny rekultywacji leśnej na wybranych terenach pogórniczych w Polsce. Wydawnictwo UR Kraków, Kraków.

Pietrzykowski, M., 2014. Soil quality index as a tool for Scots pine (Pinus sylvestris) monoculture conversion planning on afforested, reclaimed mine land. Journal of Forestry Research 25, 63-74. https:// doi.org/10.1007/s11676-013-0418-x

Pietrzykowski, M., Krzaklewski, W., 2007. Soil organic matter, C and N accumulation during natural succession and reclamation in an opencast sand quarry (southern Poland). Archives of Agronomy and Soil Science 53(5), 473-483. https://doi.org/10.1080/03650340701362516

Pietrzykowski, M., Daniels, W.L., 2014. Estimation of carbon sequestration by pine (Pinus sylvestris L.) ecosystems developed on reforested postmining sites in Poland on differing mine soil substrates. Ecological Engineering 73, 209-218. https://doi.org/10.1016/j.ecoleng.2014.09.058

Pietrzykowski, M., Krzaklewski, W., 2009. Rekultywacja leśna terenów wyrobisk po eksploatacji piasków podsadzkowych na przykładzie kopalni “Szczakowa”. Monografia. Uniwersytet Rolniczy w Krakowie, Wydział Leśny, Katedra Ekologii Lasu, Kraków.

Rowland, S.M., Prescott, C.E., Grayston, S.J., Quideau, S.A., Bradfield, G.E., 2009. Recreating a Functioning Forest Soil in Reclaimed Oil Sands in Northern Alberta: An Approach for Measuring Success in Ecological Restoration. Journal of Environmental Quality 38(4), 1580-1590. https://doi.org/10.2134/jeq2008.0317

Rumpel, C., Kögel-Knabner, I., 2003. Characterization of organic matter and carbon cycling in Rehabilitated lignite-rich mine soils. Water, Air and Soil Pollution 3(1), 153-166. https://doi.org/10.1023/ A:1022144513084
Skawina, T., 1974. Charakterystyka działalności rekultywacyjnej na zwałowisku zewnętrznym Kopalni Siarki „Piaseczno”. Instytut Kształtowania i Ochrony Środowiska AGH Kraków, maszynopis.

Solon, J., Borzyszkowski, J., Bidłasik, M., Richling, A., Badora, K., Balon, J., Brzezińska-Wójcik, T., Chabudziński, Ł., Dobrowolski, R., Grzegorczyk, I., Jodłowski, M., Kistowski, M., Kot, R., Krąż, P., Lechnio, J., Macias, A., Majchrowska, A., Malinowska, E., Migoń, P., Myga-Piątek, U., Nita, J., Papińska, E., Rodzik, J., Strzyż, M., Terpiłowski, S., Ziaja, W., 2018. Physico-geographical mesoregions of Poland: Verification and adjustment of boundaries on the basis of contemporary spatial data. Geographia Polonica 91(2), 143-170. https://doi.org/10.7163/ GPol.0115

Strzyszcz, Z., Harabin, Z., 2004. Rekultywacja i biologiczne zagospodarowanie odpadów górnictwa węgla kamiennego ze szczególnym uwzględnieniem centralnych zwałowisk. Instytut Podstaw Inżynierii Środowiska PAN, Zabrze.

Vindušková, O., Frouz, J., 2013. Soil carbon accumulation after open-cast coal and oil shale mining in Northern Hemisphere: a quantitative review. Environmental Earth Sciences 69, 1685-1698. https://doi. org/10.1007/s12665-012-2004-5

Węgorek, T., 2003. Zmiany niektórych właściwości materiału ziemnego i rozwój fitocenoz na zwałowisku zewnętrznym kopalni siarki w wyniku leśnej rekultywacji docelowej. Rozprawy Naukowe Akademii Rolniczej w Lublinie. Wydział Rolniczy. Zeszyt 275, 1-140.

Woś, B., Pietrzykowski, M., Józefowska, A., 2018. Reclaimed mine soil substrates and tree stands vs. successional forest floor vegetation: A case study of developing ecosystems on afforested mine sites. Ecological Engineering 120, 504-512. https://doi.org/10.1016/j.ecoleng.2018.07.010

Ziemnicki, S., 1980. Rekultywacja zwału kopalni odkrywkowej (na przykładzie Piaseczna). Problemy Rejonów Uprzemysławianych, Warszawa.

\section{Słowa kluczowe}

Technosole

Industriosole

Rekultywacja

Zapas węgla

$\mathrm{pH}$ gleby

\section{Właściwości gleb technogenicznych kształtujących się z substratów neogeńskich i czwartorzędowych na zrekultywowanych terenach po odkrywkowej eksploatacji siarki i piasków podsadzkowych}

\section{Streszczenie}

W pracy przedstawiono zróżnicowanie właściwości gleb technogenicznych na dwóch obiektach pogórniczych: zrekultywowanych i zalesionych zwałowisku po odkrywkowej kopalni siarki i wyrobisku piasków podsadzkowych zbudowanych odpowiednio z glin i piasków czwartorzędowych, iłów Neogeńskich (Krakowieckich) oraz mieszanin czwartorzędowych piasków z iłami Neogeńskimi. Badane gleby pogórnicze istotnie różniły się nie tylko uziarnieniem, ale także pH, właściwościami kompleksu sorpcyjnego oraz zawartością węgla i azotu. Obserwowane zakwaszenie i wzrost zawartości węgla i azotu w wierzchnich poziomach glebowych w porównaniu do poziomów skały macierzystej wskazywały na zaznaczające się coraz silniej oddziaływanie gromadzonej pod drzewostanami materii organicznej. Efekt zakwaszenia nie ujawnił się tak wyraźnie jedynie na glebach tworzących się na iłach, ze względu na ich znaczne zdolności buforowe. Jednakże, gleby wykształcające się z iłów Neogeńskich charakteryzowały się jednak największym zapasem węgla organicznego, związanego nie tylko z akumulacją in situ, ale także z zawartością geogenicznego węgla w bituminach iłów. Mniejszym zapasem węgla charakteryzowały się powstające gleby pogórnicze w kolejności na mieszaninach czwartorzędowych piasków z neogeńskimi iłami, z glin i piasków czwartorzędowych. Stwierdzane wartości większego zakwaszenia oraz większej zawartości C i N w wierzchnich poziomach glebowych w porównaniu do poziomów skały macierzystej świadczą o postępującym procesie glebotwórczym w badanych glebach pogórniczych, zainicjowanym w wyniku przeprowadzonej rekultywacji leśnej. 